\title{
Neurosonography and pathology in the Schinzel-Giedion syndrome
}

\author{
Alexander C Maclennan, David Doyle, Robert M Simpson
}

\begin{abstract}
We describe a case of the Schinzel-Giedion syndrome, where increasing ventriculomegaly, intraventricular bands, and subependymal pseudocysts were shown by ultrasound of the head. Subsequent neuropathology confirmed the hydrocephalus.
\end{abstract}

The Schinzel-Giedion syndrome is a rare form of lethal dwarfism..$^{1-3}$ Apart from physical abnormalities, these infants show gross neurological dysfunction. We report a further case, detailing the appearance of the brain on ultrasound and at necropsy.

\section{Case report}

This was the first pregnancy of an unmarried, healthy, 18 year old woman. The parents were unrelated. The mother was unsure of the date of her last menstrual period. At booking for antenatal care, the fetus was 34 to 35 weeks' gestation by ultrasound scan and there was polyhydramnios. Ten days later a baby girl of unusual appearance was born in good condition.

The infant was markedly dysmorphic (fig 1). The facies were coarse with full cheeks and plentiful facial hair. The skull was brachycephalic with widely spaced sutures and a prominent forehead. The eyes were also widely spaced with periorbital oedema and a mongoloid slant. There was a snub nose with anteverted nostrils and a short philtrum. The ears were abnormally shaped and low set. The neck was very short. There were hypoplastic nipples, but

Department of Paediatrics, Dumfries and Galloway Royal Infirmary, Dumfries DG1 4AP.

A C Maclennan, $R$ M Simpson

Department of Neuropathology, Institute of Neurological Sciences, Southern General Hospital, Glasgow G51 4TF. D Doyle

Correspondence to Dr Maclennan, Radiology Department, Glasgow Royal Infirmary, 16 Alexandra Parade, Glasgow G31 2ER.

Received for publication 6 August 1990

Revised version accepted for publication 31 December 1990. otherwise the chest, heart, and abdominal examination was normal. A deep interlabial sulcus was noted, continuous with the fold of the buttock. Her limbs were short with very small hands and feet. There were bilateral simian creases. Head circumference at $31 \mathrm{~cm}$ and weight at $2100 \mathrm{~g}$ were on the third centile. Length at $39 \mathrm{~cm}$ was markedly below the third centile. The estimated gestation was 36 weeks by appearance, agreeing with the antenatal scan.

Apart from initial hypoglycaemia (lowest blood sugar $1.1 \mathrm{mmol} / 1$ at 10 hours) routine haematology and biochemistry were normal. Chromosome analysis showed a normal female karyotype by Giemsa banding. Head ultrasound showed moderate dilatation of the third and lateral ventricles. The lateral ventricles also contained echogenic bands and there were large

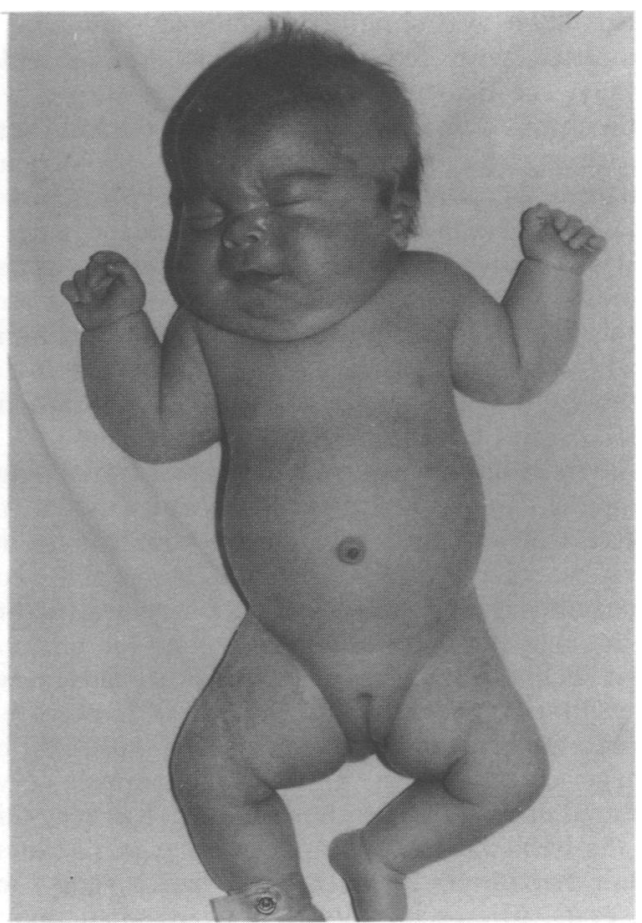

Figure 1 Patient at 3 weeks. Note dwarfism, hypertrichosis, and snub nose. 


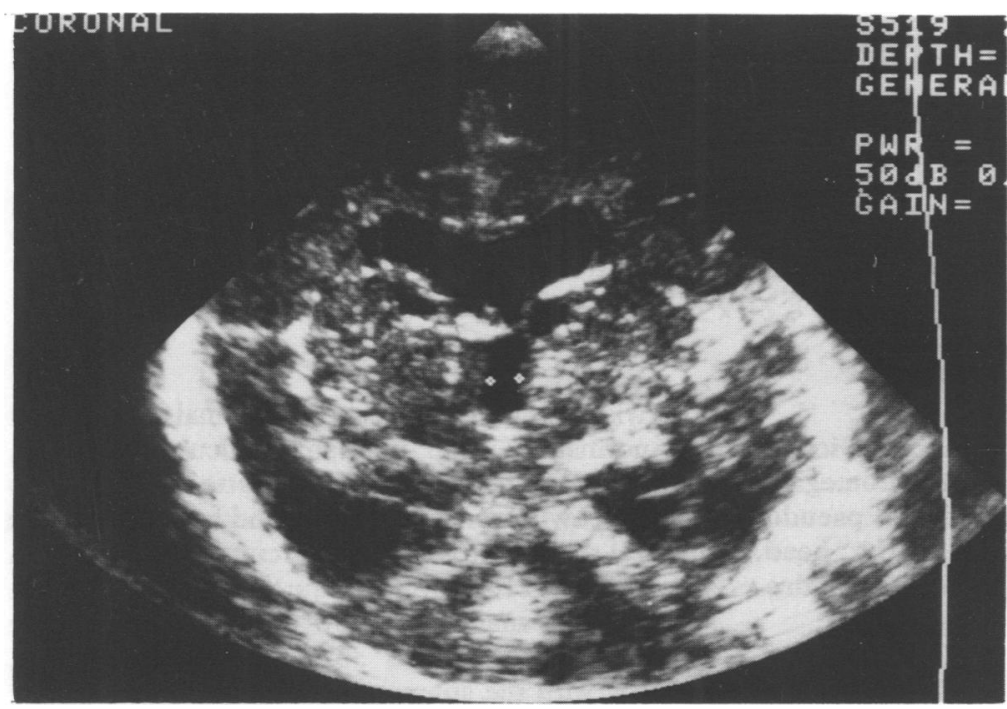

Figure 2 Coronal head ultrasound at 1 week. Note ventriculomegaly with pseudocyst formation and echogenic bands within the lateral ventricles.

subependymal cavities on the lateral aspects of the anterior horns of the lateral ventricles and by both thalamic regions (fig 2). Renal ultrasound showed moderate dilatation of the left pelvicaliceal system.

A systolic murmur developed without cardiac failure. She was briefly discharged, only to be readmitted with recurrent apnoeic attacks. Additionally, she made no developmental progress, with neither smile nor reaction to external stimuli. Serial neurosonography showed progressive biventricular dilatation but decreasing cyst sizes. She died at 9 weeks of age after a prolonged apnoeic episode.

At necropsy, the heart, great vessels, and pericardium were normal, but there was a large atrial septal defect. The alimentary system and endocrine glands appeared normal. There was a left hydronephrosis. The uterus was hypoplastic with flattened streak ovaries but normal Fallopian tubes.

The fixed brain weighed $420 \mathrm{~g}$. The leptomeninges and gyral pattern were basically normal though the insulae were incompletely closed with the frontal operculae being short. The orbital surfaces of the base of the brain were asymmetrical, with normal structures on the right, but no left gyrus rectus or olfactory nerve. The fourth nerves were narrow threads, as was the right sixth nerve. Only the left fifth nerve was present though there may have been post mortem damage on the right. The lower cranial nerves appeared normal. The corpus callosum was very thin, but the basal ganglia appeared normal. In the lateral wall of the lateral ventricles, there were several 2 to 3 mm cysts. Microscopically these were small cavities lined by stretched ependymal cells, some containing blood cells. The gliotic subependymal layer also contained islands of submerged ependyma and small cavities which appeared to be lined by endothelium. The shape of the ventricles and the absence of ependyma from large areas of ventricular walls suggested that hydrocephalus had damaged the ventricular walls. The aqueduct had numerous branches, but there was no continuity between the upper and lower parts. The brain stem was grossly normal apart from the cranial nerve abnormalities, and microscopy showed no disturbance in nerve groupings or cranial nerve nuclei.

\section{Discussion}

We believe that the physical features, clinical course, and necropsy findings confirm this to be a case of Schinzel-Giedion syndrome. Although the most impressive feature of these infants is their profound neurodevelopmental delay, little is known about their nervous system. A CT scan has shown moderate biventricular dilatation with grossly normal cerebral structures. ${ }^{3}$ The only necropsy showed abnormal cerebral gyration with subdural and subarachnoid haemorrhages. ${ }^{1}$

The obvious features, on initial head ultrasound examination of this infant, were echogenic bands within dilated ventricles. These appearances have been reported in cases of ventriculitis. ${ }^{4}$ Periventricular cavities or pseudocysts may follow an intraventricular haemorrhage or a ventriculitis, but when present at birth they suggest an intrauterine cause. They are often associated with other malformations, both cardiac and renal. ${ }^{6}$ 
Pathological examination of the brain confirms the ventricular enlargement, with discontinuity of the aqueduct. The pseudocysts are small, as expected from scanning. There is no histological evidence of an infective or inflammatory process.

The Schinzel-Giedion syndrome is probably of autosomal recessive inheritance. ${ }^{1}$ We agree that the neuropathology suggests a fundamental genetic abnormality. Unfortunately, the reason for the extreme neurodevelopmental delay remains unclear, as the degree of handicap was much greater than expected from the ventriculomegaly.
1 Schinzel A, Giedion A. A syndrome of severe midface retraction multiple skull anomalies, clubfeet, and cardiac and renal malformation in sibs. Am $\mathcal{F}$ Med Genet 1978;1:361-75.

2 Donnai $D$, Harris $R$. A further case of a new syndrome including midface retraction, hypertrichosis, and skeletal anomalies. f Med Genet 1979;16:483-6.

3 Kelley RI, Zackai EH, Charney EB. Congenital hydronephrosis, skeletal dysplasia, and severe developmental retardation: the Schinzel-Giedion syndrome. $\mathcal{F}$ Pediatr 1982;100:943-6.

4 Edwards MK, Brown DL, Chua GT. Complicated infantile meningitis: evaluation by real time sonography. Am $\mathcal{f}$ Neuroradiol 1982;3:431-4.

5 Reeder JD, Sanders RC. Ventriculitis in the neonate: recognition by sonography. Am $\mathcal{F}$ Neuroradiol 1983;4:37-41.

6 Larroche JC. Sub-ependymal pseudocysts in the newborn. Biol Neonate 1972;21:170-83. 